\title{
Multivariate Statistics of the Jacobian Matrices in Tensor Based Morphometry and Their Application to HIV/AIDS
}

\author{
Natasha Lepore $^{1}$, Caroline A. Brun ${ }^{1}$, Ming-Chang Chiang ${ }^{1}$, Yi-Yu Chou ${ }^{1}$, \\ Rebecca A. Dutton ${ }^{1}$, Kiralee M. Hayashi ${ }^{1}$, Oscar L. Lopez ${ }^{2}$, \\ Howard J. Aizenstein ${ }^{3}$, Arthur W. Toga ${ }^{1}$, \\ James T. Becker ${ }^{2}$, and Paul M. Thompson ${ }^{1}$ \\ ${ }^{1}$ Laboratory of Neuro Imaging, Department of Neurology, David Geffen School of \\ Medicine at UCLA, Los Angeles, CA 90095 USA \\ ${ }^{2}$ Department of Psychiatry, University of Pittsburgh, Pittsburgh, PA 15213 USA \\ ${ }^{3}$ Department of Neurology, University of Pittsburgh, Pittsburgh, PA 15213 USA*
}

\begin{abstract}
Tensor-based morphometry (TBM) is widely used in computational anatomy as a means to understand shape variation between structural brain images. A 3D nonlinear registration technique is typically used to align all brain images to a common neuroanatomical template, and the deformation fields are analyzed statistically to identify group differences in anatomy. However, the differences are usually computed solely from the determinants of the Jacobian matrices that are associated with the deformation fields computed by the registration procedure. Thus, much of the information contained within those matrices gets thrown out in the process. Only the magnitude of the expansions or contractions is examined, while the anisotropy and directional components of the changes are ignored.
\end{abstract}

Here we remedy this problem by computing multivariate shape change statistics using the strain matrices. As the latter do not form a vector space, means and covariances are computed on the manifold of positivedefinite matrices to which they belong. We study the brain morphology of $26 \mathrm{HIV} / \mathrm{AIDS}$ patients and 14 matched healthy control subjects using our method.

The images are registered using a high-dimensional 3D fluid registration algorithm, which optimizes the Jensen-Rényi divergence, an information-theoretic measure of image correspondence. The anisotropy of the deformation is then computed. We apply a manifold version of Hotelling's $T^{2}$ test to the strain matrices. Our results complement those found from the determinants of the Jacobians alone and provide greater power in detecting group differences in brain structure.

\footnotetext{
* This research was supported by the National Institute on Aging (AG021431 and AG016570), the National Library of Medicine, the National Institute for Biomedical Imaging and Bioengineering, the National Center for Research Resources (LM05639, EB01651, RR019771), and a Research Scientist Development Award - Level II (MH01077).
} 


\section{Introduction}

Accurate measurement of differences in brain anatomy is key to understanding the effects of brain disorders, as well as changes associated with normal growth or variation within a population. Statistical methods that make full use of the available data can greatly improve the analysis of temporal changes within a single subject, as well as inter-subject variations. Given the subtle and distributed brain changes that occur with development and disease, improved statistical analyses are needed that detect group differences or time-dependent changes in brain structure with optimal power.

In tensor-based morphometry (TBM), a template $T$ is matched to a study $S$ using non-linear registration, and the displacement vector $\boldsymbol{u}(\boldsymbol{r})$ is found such that $T(\boldsymbol{r}-\boldsymbol{u})$ corresponds with $S(\boldsymbol{r})$. Here $\boldsymbol{r}$ denotes the voxel location. To help estimate anatomical correspondences, features such as point, curve, and surface landmarks present in both datasets can be used, or, more commonly, intensity-based cost functions are used based on normalized cross-correlation, mean square intensity difference, or mutual information. The Jacobian matrix of the deformation field is defined (in 3D) by

$$
J=\left(\begin{array}{l}
\partial\left(x-u_{x}\right) / \partial x \partial\left(y-u_{y}\right) / \partial x \partial\left(z-u_{z}\right) / \partial x \\
\partial\left(x-u_{x}\right) / \partial y \partial\left(y-u_{y}\right) / \partial y \partial\left(z-u_{z}\right) / \partial y \\
\partial\left(x-u_{x}\right) / \partial z \partial\left(y-u_{y}\right) / \partial z \partial\left(z-u_{z}\right) / \partial z
\end{array}\right)
$$

Its determinant, the Jacobian, is most commonly used to analyze the distortion necessary to deform the images into agreement. A value $\operatorname{det} J(\boldsymbol{r})>1$ implies that the neighborhood adjacent to $\boldsymbol{r}$ in the study was stretched to match the template (i.e., local volumetric expansion), while $\operatorname{det} J(\boldsymbol{r})<1$ is associated with local shrinkage. When many subjects' images are aligned to the same standard template or atlas, maps of the Jacobians can be computed in the atlas coordinate system and group statistics can be computed at each voxel to identify localized group differences in anatomical shape or size.

However, much of the information about the shape change is lost using this measure. As a toy example of this problem, let us consider a pixel for which the eigenvalues of the Jacobian are $\lambda_{1,2,3}=\{1,2,0.5\}$. In such a case, the value of the Jacobian would be 1 . Thus, though the eigenvalues clearly indicate directional shrinkage and growth, these changes would be left undetected, if only the Jacobian were analyzed.

In this work, we resolve this issue by making use of the strain matrices for the analysis, which are defined as $\left(J^{T} J\right)^{1 / 2}$. In particular, we apply Hotelling's $T^{2}$ test to obtain statistics for the deformation.

However, the problem is complicated by the fact that for smooth transformations, the strain matrices are constrained to the space of positive definite matrices. The latter form a cone in the space of matrices, which is itself a vector space. Thus, the strain matrices do not form a vector space, and a manifold version of the statistics is needed.

Several research groups have worked on the statistics of positive definite symmetric matrices. The first person to investigate this problem was Fréchet [1], who 
defined the mean $\bar{S}$ on a manifold as the point that leads to a minimum value for the sum of squared geodesic distances between $\bar{S}$ and all the other points. $\overline{(} S$ ) yields the usual value for the mean in Euclidean space, but is not necessarily unique on other manifolds. Thus, the local value of the mean is generally used instead [2, 3]. To facilitate computations, Pennec et al. 44, 5] and Fletcher and Joshi [6] independently proposed the use of the affine-invariant metric on the space of symmetric, positive-definite tensors. Pennec then proceeded to use the latter to define normal distributions on these matrices, as well as the Mahalanobis distance and $\chi^{2}$ law. Fletcher and Joshi 6, 6] used the metric to create the principal geodesic analysis, which is an extension of principal component analysis to manifolds. The application of these techniques to calculate means of Jacobian matrices was first suggested by Woods [8]. In 9], statistics on strain tensors were used to determine the a weight factor for the regularizer in non-rigid registration. Furthermore, the Mahalanobis distance of these tensors was used as an elastic energy for the regularizer in [10. However, to our knowledge this is the first time the full matrices are used in the context of tensor-based morphometry.

Once a metric is defined, the manifold-valued elements are projected into the tangent plane at the origin using the inverse of the exponential map, and the computations are done in this common space. Recently, Arsigny et al. [11, considered a new family of metrics, the 'Log-Euclidean metrics'. The latter facilitate computations, as they are chosen such that the transformed values form a vector space, and thus statistical parameters can be easily computed using standard formulae for Euclidean spaces. For two points $S_{1}$ and $S_{2}$ on the manifold, these metrics are of the form

$$
d\left(S_{1}, S_{2}\right)=\left\|\log S_{1}-\log S_{2}\right\|
$$

where $\|$.$\| denotes a norm. Following [11], in this work we will use,$

$$
d\left(S_{1}, S_{2}\right)=\left(\operatorname{Trace}\left(\log S_{1}-\log S_{2}\right)^{2}\right)^{1 / 2} .
$$

In this paper, we use a set of brain MRI scans from HIV/AIDS patients and matched healthy control subjects to illustrate our method. We use a fluid registration algorithm [12] to compute displacement fields and thus obtain strain matrices. All our statistics are computed within the Log-Euclidean framework. A pixelwise Hotelling $T^{2}$ test is used as a measure of variation between patients and controls. To assess the difference between our results and the ones found from the determinant of the Jacobian, these results are compared to the onedimensional Student's t test on the determinant of the Jacobian matrices. The goal of the work was to find out whether multivariate statistics on the strain tensor afforded additional power in detecting anatomical differences between patients and controls.

The usual measure of anisotropy for tensor-valued data is the fractional anisotropy (used widely in diffusion tensor imaging) but this measure is not valid on positive definite symmetric manifolds, as it relies on a Euclidean measure for the distance. Thus, in [13, the authors propose a new measure which depends instead solely on distances computed on the manifold. They define the 
geodesic anisotropy as the shortest geodesic distance between the tensor and the closest isotropic tensor within the affine-invariant metric framework. Similarly, in the Log-Euclidean metric, we can define a geodesic anisotropy $G A$ as:

$$
G A(S)=\left(\operatorname{Trace}\left(\log S-(\langle\log S\rangle I)^{2}\right)^{1 / 2}, \quad \text { with }\langle\log S\rangle=\operatorname{Trace}(\log S) / 3 .\right.
$$

\section{Method}

Twenty-six HIV/AIDS patients (age: $47.2 \pm 9.8$ years; $25 \mathrm{M} / 1 \mathrm{~F} ; \mathrm{CD}^{+}{ }^{+}$T-cell count: $299.5 \pm 175.7$ per $\mu \mathrm{l} ; \log _{10}$ viral load: $2.57 \pm 1.28 \mathrm{RNA}$ copies per $\mathrm{ml}$ of blood plasma) and $14 \mathrm{HIV}$-seronegative controls (age: $37.6 \pm 12.2$ years; $8 \mathrm{M} / 6 \mathrm{~F}$ ) underwent 3D T1-weighted MRI scanning; subjects and scans were the same as those analyzed in the cortical thickness study by Thompson et al. 14, where more detailed neuropsychiatric data from the subjects is presented. All patients met Center for Disease Control criteria for AIDS, stage C and/or 3 (Center for Disease Control and Prevention, 1992), and none had HIV-associated dementia. Health care providers in Allegheny County, PA, served as a sentinel network for recruitment. All AIDS patients were eligible to participate, but those with a history of recent traumatic brain injury, CNS opportunistic infections, lymphoma, or stroke were excluded.

All patients underwent a detailed neurobehavioral assessment within the 4 weeks before their MRI scan, involving a neurological examination, psychosocial interview, and neuropsychological testing, and were designated as having no, mild, or moderate (coded as 0,1 , and 2 respectively) neuropsychological impairment based on a factor analysis of a broad inventory of motor and cognitive tests performed by a neuropsychologist 14.

All subjects received 3D spoiled gradient recovery (SPGR) anatomical brain MRI scans $(256 \times 256 \times 124$ matrix, $\mathrm{TR}=25 \mathrm{~ms}, \mathrm{TE}=5 \mathrm{~ms} ; 24$ - $\mathrm{cm}$ field of view; 1.5 $\mathrm{mm}$ slices, zero gap; flip angle $=40^{\circ}$ ) as part of a comprehensive neurobehavioral evaluation. The MRI brain scan of each subject was co-registered with scaling (9parameter transformation) to the ICBM53 average brain template, after removal of extracerebral tissues (e.g., scalp, meninges, brainstem and cerebellum).

The images were non-linearly registered using a fluid registration algorithm [12, based on a convolution filter developed by Bro-Nielsen and Gramkow [15, [16 to increase the speed of the registration. As a cost function, we chose to maximize a modified version of the Jensen-Rényi Divergence (JRD). A more detailed description of our registration method can be found in [17]. To save computation time and memory requirements, the source and the target images were filtered with a Hann-windowed sinc kernel, and isotropically downsampled by a factor of 2 . As in other TBM studies [18, 19, we preferred registration to a typical control image rather than a multi-subject average intensity atlas as it had sharper features. The resulting deformation field was trilinearly interpolated to drive the source image towards the target at the original resolution to obtain the warped image.

The geodesic anisotropy $G A$ was found at each voxel using eq.2 $\tanh (G A)$ is displayed in Fig.1 a) and b). The hyperbolic tangent of the geodesic anisotropy 
was used rather than $G A$ itself, as it takes values in the interval $[0,1]$, while those of $G A$ span the interval $[0, \infty)$ (see [13]). We notice widespread directionality in the strain matrices, which strengthens the idea that valuable information may be found from the non-uniform components of the changes.

In order to apply Hotelling's $T^{2}$ test, we need to compute the mean and covariance matrices of the tensor-valued data. Thus, here we provide a brief summary of the method to find those quantities.

In $R^{n}$, the mean $\bar{S}$ of a set of n-dimensional vectors $S_{i}, i=1, \ldots, m$ is the point that minimizes the summed squared distance $d$ to all the $S_{i}$. For data on a manifold $A, d$ becomes the geodesic distance, so $\bar{S}$ is given by [1]:

$$
\bar{S}=\operatorname{argmin}_{S \epsilon A} \sum_{i=1}^{m} d\left(S, S_{i}\right)^{2} .
$$

In the log-Euclidean framework, computations are simplified by transforming the space of symmetric, positive-definite matrices into a vector space on the tangent plane, and then transforming it back to the manifold using the exponential map once the mean is taken. Thus, the formula for the mean is easily shown to be [1] :

$$
\bar{S}=\exp \left(\frac{1}{M} \Sigma_{i=1}^{m} \log S_{i}\right)
$$

Arsigny et al. demonstate that the covariance is

$$
\operatorname{Cov}(S)=\int_{\Omega}(\log (S(w))-\log (\bar{S}(w))) \otimes(\log (S(w))-\log (\bar{S}(w))) d P(w),
$$

where $\mathrm{dP}$ is the probability measure, $\Omega$ is the space of possible outcomes and $w \epsilon \Omega$. For discrete data, this becomes

$$
\bar{C}=\frac{1}{M} \Sigma_{i=1}^{M}(\log S-\log \bar{S})(\log S-\log \bar{S})^{T}
$$

Thus, we obtain the Mahalanobis distance

$$
M=(\log S-\log \bar{S}) \Sigma^{-1}(\log S-\log \bar{S})^{T}
$$

So as to not assume a normal distribution, we performed a voxelwise permutation test, for which we randomly reassigned the labels of patients and controls, and compared the p-values so generated to those of the data. All our statistics were computed using 5000 permutations.

\section{$3 \quad$ Results}

Fig. 1 c) shows the p-values from the Student's t-test for the $\log _{10} J$ distribution. The corresponding values for Hotelling's $T^{2}$ test are shown in Fig.1 d). The p-values are considerably lower in the case of Hotelling's $T^{2}$ test. The latter is thus more sensitive, and can detect differences which are not detected with 
the conventional method, even when operating on the same deformation fields. Group differences in brain structure between AIDS patients and healthy subjects are visible throughout the brain, with the greatest effect sizes in the corpus callosum and basal ganglia. That result is confirmed by prior TBM studies of HIV/AIDS [17. The white matter also exhibits widespread atrophy, which former neuropathology studies on HIV/AIDS have shown. The cortical region is noisier, perhaps because the registration method is intensity-based and does not perform as well in that area.
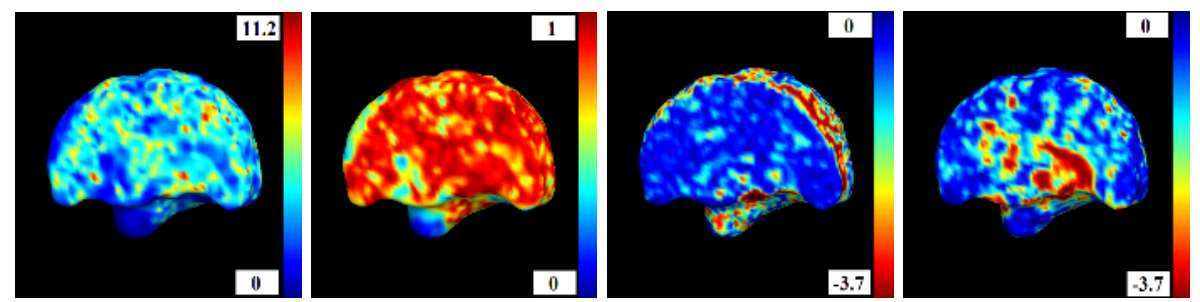

Fig. 1. From left to right: a) Voxelwise geodesic anisotropy calculated from eq2 b) Its hyperbolic tangent c) Voxelwise p-values given by the Student's t-test for the $\log _{10}(J)$. d) Voxelwise p-values computed from the Hotelling $T^{2}$ test on the strain matrices. P-values are shown on a $\log _{10}$ scale; -3 denotes a voxel level p-value of 0.001 . A sagittal section of the brain is shown, with the brain facing to the left. As expected from the literature on the neuropathology of HIV/AIDS, greatest atrophic effects are found in the subcortical regions, which border on ventricular regions enriched in the virus. The Lie group methods show comparable patterns of atrophy to the standard method, but with much greater sensitivity (i.e., better signal to noise).

\section{Conclusions}

Here we used a combination of tensor-based morphometry, Lie group methods, and multivariate statistics to detect systematic differences in anatomy between $\mathrm{HIV} / \mathrm{AIDS}$ patients and controls. The anatomical profile of group differences is in line with studies using traditional volumetric methods, as the HIV virus is known to cause widespread neuronal loss and corresponding atrophy of the gray and white matter, especially in subcortical regions. What is more surprising is this multivariate method shows that finding with a much greater power than conventional TBM. In TBM, the Jacobian of the deformation is commonly examined, and multiple regression is applied to the scalar data from all subjects at each voxel, to identify regions of volumetric excess or deficit in one group versus another. This is clinically relevant especially given the large efforts, for example in drug trials of neurodegenerative disease, to detect subtle effects of disease on the brain using minimal numbers of subjects (or the shortest possible follow-up intervals, in a longitudinal imaging study). As such it is important to optimize not only the nonlinear registration methods that gauge alterations in brain structure, but also the statistical methods for analyzing the resulting tensor-valued 
data. The current work can be expanded in several ways. First, in a multivariate setting, deformation fields could be analyzed using differential operators or tensors other than the strain tensor or deformation gradient; Rey and Cachier 20], Ashburner 21], Leow 22] and others have proposed examining the Hencky strain tensors, the logarithm of the scalar Jacobian. The optimal tensor to use for detecting group differences in TBM may depend on the directional properties of the underlying disease or developmental process being analyzed, as well as the differential operator used to regularize the deformation. Analytic formulae for the null distribution of the volume of the excursion sets of $T^{2}$-distributed random fields were recently computed by Cao and Worsley [23] 24], and these may also be applied in a Lie group setting to optimize signal detection. Studies are also underway to incorporate the log-Euclidean framework in the cost functions (or statistical priors) that regularize the nonlinear registrations themselves. This suggests that Lie group methods may be advantageous for both the registration and statistical analysis of tensor-valued data.

\section{References}

1. Fréchet, M.: Les éléments aléatoires de nature quelconque dans un espace distancié. Ann. Inst. H. Poincaré 10 (1948) 215-310

2. Karcher, H.: Riemannian center of mass and mollifier smoothing. Commun Pure Appl. Math. 30 (1977) 509-541

3. Kendall, W.S.: Probability, convexity and harmonic maps with small imageI: Uniqueness and fine existence. Proc. Lond. Math. Soc. 3 (1990) 371406

4. Pennec, X.: Probabilities and statistics on Riemannian manifolds: Basic tools for geometric measurements. In: Proceedings of the IEEE-EURASIP Workshop on Non-Linear Signal and Image Processing (NSIP99), Antalya, Turkey (1999) 194-198

5. Pennec, X., Fillard, P., Ayache, N.: A Riemannian framework for tensor computing. International Journal of Computer Vision 66 (2006) 4166 A preliminary version appeared as INRIA Research Report 5255, July 2004.

6. Fletcher, P.T., Joshi, S.: Principal geodesic analysis on symmetric spaces: Statistics of diffusion tensors. In: Computer Vision ECCV 2004, Prague, Czech Republic (2004) 87-98

7. Fletcher, P.T.: Statistical Variability in Nonlinear Spaces: Application to Shape Analysis and DT-MRI. PhD thesis, Department of Computer Science, University of North Carolina, Chapel Hill, NC (2004)

8. Woods, R.P.: Characterizing volume and surface deformation in an atlas framework: theory, applications and implementation. NeuroImage 18 (2003) 769-788

9. Commowick, O., Stefanescu, R., Fillard, P., Arsigny, V., Ayache, N., Pennec, X., Malandain, G.: Incorporating statistical measures of anatomical variability in atlas-to-subject registration for conformal brain radiotherapy. In: Proceedings 8th International Conference on Medical Image Computing and Computer-Assisted Intervention (MICCAI), Part II, Palm Springs, CA, USA (2005) 927-934

10. Pennec, X., Stefanescu, R., Arsigny, V., Fillard, P., Ayache, N.: Riemannian elastic- ity: A statistical regularization framework for non-linear registration. In: Proceedings 8th International Conference on Medical Image Computing and Computer- Assisted Intervention (MICCAI), Palm Springs, CA, USA (2005) 943-950 
11. Arsigny, V., Fillard, P., Pennec, X., Ayache, N.: Log-Euclidean metrics for fast and simple calculus on diffusion tensors. Magnetic Resonance in Medicine (2006) To appear. Preprint: INRIA RR 5584, 2005.

12. Christensen, G., Rabbitt, R., Miller, M.: Deformable templates using large deformation kinematics. IEEE Trans. Image Process. 5 (1996) 14351447

13. Batchelor, P., Moakher, M., Atkinson, D., Calamante, F., Connelly, A.: A rigorous framework for diffusion tensor calculus. Magnetic Resonance in Medicine 53 (2005) $221-225$

14. Thompson, P., Dutton, R., Hayashi, K., Toga, A., Lopez, O., Aizenstein, H., Becker, J.: Thinning of the cerebral cortex visualized in HIV/AIDS reflects CD4+Tlymphocyte decline. Proc. Natl. Acad. Sci. USA 102 (2005) 1564752

15. Bro-Nielsen, M., Gramkow, C.: Fast fluid registration of medical images. In: Proceedings of the 4th International Conference on Visualization in Biomedical Computing, Hamburg, Germany (1996) 267-276

16. Gramkow, C.: Registration of 2D and 3D medical images. Masters thesis, Danish Technical University, Copenhagen, Denmark (1996)

17. Chiang, M.C., Dutton, R.A., Hayashi, K.M., Lopez, O.L., Aizenstein, H.J., Toga, A.W., Becker, J.T., Thompson, P.M.: 3D pattern of brain atrophy in HIV/AIDS visualized using tensor-based morphometry. NeuroImage submitted (2006)

18. Studholme, C., Cardenas, V., Schuff, N., Rosen, H., Miller, B., Weiner, M.: Detecting spatially consistent structural differences in Alzheimers and fronto temporal dementia using deformation morphometry. In: Proceedings of International Conference onMedical Image Computing and Computer Assisted Intervention (MICCAI), Utrecht, Netherlands (2001) 41-48

19. Davatzikos, C., Genc, A., Xu, D., Resnick, S.: Voxel-based morphometry using the RAVENS maps: Methods and validation using simulated logitudinal atrophy. NeuroImage 14 (2001) 1361-1369

20. Cachier, P., Rey, D.: Symmetrisation of the non-rigid registration problem using inversion-invariant energies: Application to multiple sclerosis. In: Proceedings, MICCAI 2000. Volume 1935., Pittsburgh, PA (2000) 472-481

21. Ashburner, J., Friston, K.: High-dimensional image registration using symmetric priors. NeuroImage 9 (1999) 619-628

22. Leow, A.D., Geng, A., Becker, J., Davis, S.W., Toga, A.W., Thompson, P.M.: Inverse consistent mapping in 3D deformable image registration: Its construction and statistical properties, information processing in medical imaging. In: IPMI, Glenwood Springs, Colorado (2005)

23. Cao, J.: The excursion set of random fields with applications to human brain mapping. PhD thesis, Dpt. Mathematics and Statistics, McGill University, Montreal, Canada (1997)

24. Cao, J., Worsley, K.: Application of random fields in human brian mapping. In: M. Moore (Ed.) Spatial Statistics: Methodological Aspects and Applications. Volume 159. (2001) 169-182 